\title{
Development of Non-Restraint Blood Pressure Measurement Method Utilizing PWV
}

\author{
Yasunari Asakura \\ School of Integrative and Global Majors, University of Tsukuba, Tsukuba, Japan \\ Email: asakura@golem.kz.tsukuba.ac.jp \\ Yoshiyuki Sankai \\ Faculty of Engineering, Information and Systems, the Center for Cybernics Research, University of Tsukuba, Tsukuba, \\ Japan \\ Email: sankai@golem.kz.tsukuba.ac.jp
}

\begin{abstract}
Hypertension is a major risk factor of ischemic heart disease and cerebrovascular disease. In order to prevent hypertension, it is important to measure blood pressure regularly. Non-restraint blood pressure measurement method would make daily measurement convenience. The purpose of this study is to develop a novel non-restraint measurement method and device of PWV (Pulse Wave Velocity) that relates to blood pressure strongly and verify the feasibility of measuring PWV with the proposed method. We developed pressure plethysmogram sensors that could detect BCG (Ballistocardiogram), and attached it on a chair. PWV was calculated from two characteristic points of BCG, which were the rising point of $H$ wave and the bottom of $I$ wave. In the experiment, developed system is applied to five participants, and measured values of PWV are increased according to values of systolic blood pressure. In addition, the correlation coefficients are enough to imply the relationship between PWV measured by our device and systolic blood pressure. In conclusion, we developed a novel non-restraint measurement method and device of PWV that relates to blood pressure strongly and confirmed that correlation between systolic blood pressure and PWV by using this method and device.
\end{abstract}

Index Terms-blood pressure, pulse wave velocity (PWV), ballistocardiogram (BCG), non-restraint measurement

\section{INTRODUCTION}

Hypertension is a major factor of heart disease and cerebrovascular disease [1]. The problem is that it is difficult to detect hypertension in the early stage because hypertension has no subjective symptoms [1]. Therefore, most people do not find that they have hypertension for long term, and high blood pressure damage their blood vessels steadily. In order to detect hypertension in the early stage and to prevent it, it is essential to measure blood pressure regularly.

Measurement time and environment affect blood pressure strongly [2]. For example, some patients exhibit a blood pressure value within the normal range in the clinical settings, even though they exhibit high blood

Manuscript received May 15, 2016; revised January 12, 2017. pressure in daily life. That is called masked hypertension. On the other hand, some people have blood pressures that are higher than normal in medical settings, however their blood pressures are normal in daily life. That is called white coat hypertension. Such false measurement can lead to misdiagnosis of hypertension. In order to avoid this problem, it is required to measure blood pressure not only at a medical institution but also in daily life, and evaluate the tendency of it [3]. Such blood pressure measurement requires non-restraint measurement method and device because conventional sphygmomanometers require to squeeze an upper-arm tightly by cuff, that is discomfort for users. In addition, it takes some labor and time.

There are methods of utilizing Pulse Wave Velocity (PWV) in previous researches in order to measure blood pressure without cuff [4] - [5]. It is known that PWV and systolic blood pressure strongly correlates to each other [6]. However, conventional measurement methods of PWV are not non-restraint because those methods use electrocardiogram (ECG), photoplethysmogram (PPG) or pulse wave, which require to attach sensors such as electrodes, probes or cuffs to the body. Measuring PWV without attaching any equipment would contribute to measure blood pressure without constraint on a daily basis.

The purpose of this study is to develop a novel nonrestraint measurement method for measuring PWV, which relates to blood pressure strongly, and verify the relationship between the systolic blood pressure and PWV measured by the proposed method.

\section{MEASUREMENT METHOD}

Measurement postures and body movements affect blood pressure [7]. Therefore, it is essential to measure in seated resting posture for accurate blood pressure measurement. Embedded sensor system in chair, it is effective to measure blood pressure without constraint on a daily basis. Therefore, this study focus on a chair and attach the sensor on it for measuring PWV.

\section{A. Pulse Wave Verocity $(P W V)$}

PWV is the velocity of the pulse wave, which propagates through arteries. PWV is used as an index of 
the elasticity of the arteries in medical field. As shown in (1), PWV is defined as the length of the artery to be measured divided by Pulse Transit Time (PTT).

$$
P W V=\frac{L}{P T T}
$$

where $L$ is the propagation distance, which is the length of the artery to be focused on at the PWV measurement method, $P T T$ is the time that pulse wave takes to propagate the arteries. As shown in (2), PTT is calculated as the difference of time measured from two points of the body.

$$
P T T=t_{1}-t_{0}
$$

where $t_{0}$ is the time measured at the heart side of the artery. $t_{1}$ is the time measured at the peripheral side of the artery. Fig. 1 shows the measurement principle of PWV.

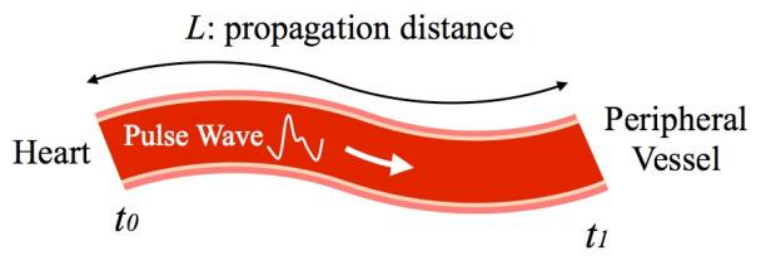

Figure 1. Schematic of the PWV measurement principle

\section{B. The Relashionship between PWV and Systolic Blood Presssure}

As shown in (3), the equation is called MoensKorteweg formula which shows the relationship between PWV and elasticity of vessels.

$$
\mathrm{PWV}=\sqrt{\frac{E \cdot h}{2 r \cdot \rho}}
$$

where $E$ is Young's modulus, which shows elasticity of a blood vessel, $h$ is the thickness of blood vessel wall, $r$ is the radius of blood vessel, $\rho$ is the density of blood.

The relationship between PWV and blood pressure is derived from the relationship between blood pressure and the surface tension of blood vessel wall caused by the change of blood pressure. In order to show that, YoungLaplace equation is used. This equation shows the relationship between pressure and surface tension in hydrodynamics field. Young-Laplace equation is defined as shown in (4).

$$
P=\mathrm{s}\left(\frac{1}{r_{1}}+\frac{1}{r_{2}}\right)
$$

where $P$ is the internal pressure, $S$ is the surface tension, $r_{1}$ and $r_{2}$ are the principal radii of curvature. Blood vessels are cylindrical, therefore, we put $r_{2}=\infty$. Then (4) is expressed as (5).

$$
P=\frac{S}{r}
$$

(6) is derived from (3) and (5).

$$
P=\frac{2 \rho S}{E h} \cdot P W V^{2}
$$

where $\mathrm{P}$ means systolic blood pressure. Consequently, systolic blood pressure is calculated from (5) by using PWV.

\section{Proposed Measurement Method}

There are several types of PWV. Major conventional index is known as cfPWV (carotid-femoral PWV), hfPWV (heart-femoral PWV), baPWV (brachial-ankle PWV) and haPWV (heart-ankle PWV) [8]. However, those methods require to attach cuffs or electrodes to the body.

When people sit on a chair, the seating surface and the body are in contact with each other. Additionally, when people take a resting and seating posture, ballistocardiogram (BCG) would be capable of measuring on the seating surface [9]. BCG is the physiological index which reflects pumping motion of heart and pulse wave [9]. Therefore, this study employs BCG in measuring PWV.

As shown in Fig. 2, there is a relationship between typical ECG waveform and typical ECG waveform. Fig. 2 also shows characteristic points of BCG, which used to calculate PWV in proposed method. These points are shown as a circle. Physiologically, the rising point of the $\mathrm{H}$ wave is caused by the movement of the heart for ejecting blood [9]. Moreover, The I wave is caused by a shock wave which the blood reaches to the aortic arch [9]. This study employs the time difference between these two points in calculating PTT.

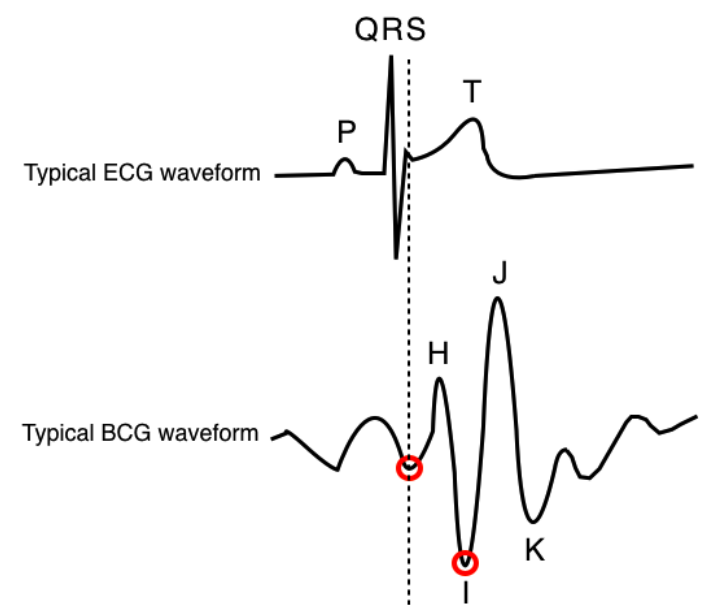

Figure 2. The relationship between the typical ECG waveform and the typical BCG waveform. Upper waveform is Typical ECG and lower one is BCG. In this figure, capital letters are the name of each characteristic wave. The dot line indicates the timing of rising point of $\mathrm{H}$ wave of BCG. In addition, circles are the characteristic points which is extracted by our method.

The length of pulse wave propagation $L$ is the length from the heart to the aortic arch. In this study, $L$ is defined $5 \mathrm{~cm}$, which is the length of the ascending aorta [10] because we are not able to measure that length noninvasively for each person. 
In this study, BCG is measured by the sensor system developed to measure minute vibration as a change of pressure. As shown in Fig. 3, The developed device consists of the measurement probe, the sensor circuit and the chair. The whole system consists of the device, a data logger (NR-2000, KEYENCE) and a Personal Computer (PC). The Measurement probe consists of the non-elastic bag filled with water, which is non-compressible fluid. Moreover, it converts vibration to a pressure change. The sensor circuit consists of the pressure sensor (XFHM200KPGR, Fujikura), the amplifier circuit and the bandpass filter circuit. The gain of the amplifier circuit is set to $40 \mathrm{~dB}$. The order of the band-pass filter is 1 and the passband is $0.05-48 \mathrm{~Hz}$. Fig. 4 shows the block diagram of developed device.

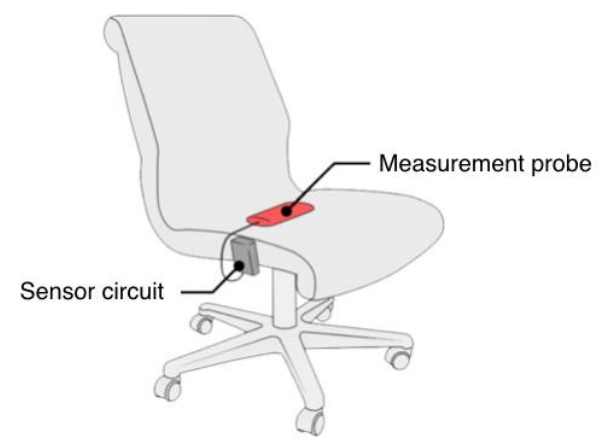

Figure 3. The position of the measurement probe and the sensor circuit.

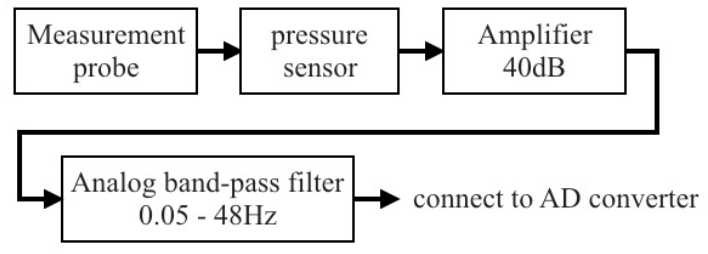

Figure 4. Block diagram of developed device

The pressure sensor measures pressure applied on the measurement probe, and analog band-pass filter processing is carried out, and that signal is amplified. The signal is performed A/D conversion, which resolution is 14-bits and sampling frequency is $1 \mathrm{kHz}$, thereafter processed data are recorded on PC. In order to avoid commercial power supply noise, all circuits, a sphygmomanometer and a PC are driven by battery.

When the user is sitting in the device, positional relation between the measurement probe and a user is shown in the Fig. 5. The measurement probe is attached to the seating surface where the position of coccyx comes in contact with that.

The measurement probe measure artifacts superimposed on the BCG because it touches to the user's body. The main component of artifact is the movement due to breathing. In order to extract the characteristic points, it is necessary to remove artifacts. The motion of the heart pumping and the artifact caused by breathing is separable because human beings' heart rate and respiration rate is clearly different. The average of heart rate is about 54 - 76 times per minute [11] and respiratory rate is about 12 - 15 times per minute [12]. The sensor system removes the component of the respiratory waveform by processing digital band-pass filter on the PC. The digital band-pass filter is processed by means of Fourier transform. The passband is $0.8-30 \mathrm{~Hz}$ so that digital filter removes respiration component. In this study, the bottom of the I wave is used to extract characteristic points because I wave is the most obvious inflection point among the characteristic point of BCG. The bottom of I wave is extracted by utilizing a threshold value. A threshold value is determined by the minimum value of the measured waveform, and the threshold value is three tenth as large as the minimum value in this study. Then, the time series data is searched backward from the bottom of I wave, and the first minimum point is the rising point of $\mathrm{H}$.

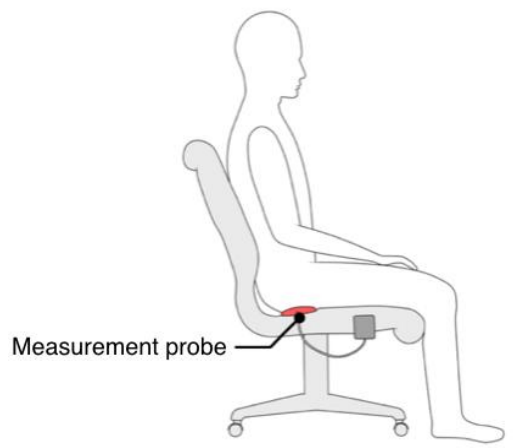

Figure 5. The positional relation between the measurement probe and the user.

In this study, ECG is used to compare with BCG in the experiment. The developed device need not to measure ECG for measuring BCG. ECG is measured by ECG measurement circuit (EVAL-AD8232, Analog Devices).

Measurement method is three-lead ECG. Electrode is wet-type electrode (Vitrode D, NIHON KOHDEN). As shown in Fig. 6 and Fig. 7, digital filter removes the artifact, and characteristic points are extracted. In Fig. 6 and Fig. 7, red-solid lines indicate BCG waveforms and blue-doted lines indicate ECG waveforms.

In addition, Fig. 6 shows measured waveforms, which artifact mainly caused by respiration. Fig. 7 shows processed waveforms which is performed by digital bandpass filter. In Fig. 7, rising points of the $\mathrm{H}$ wave is shown in circle markers and also points of $\mathrm{I}$ is shown in square markers.

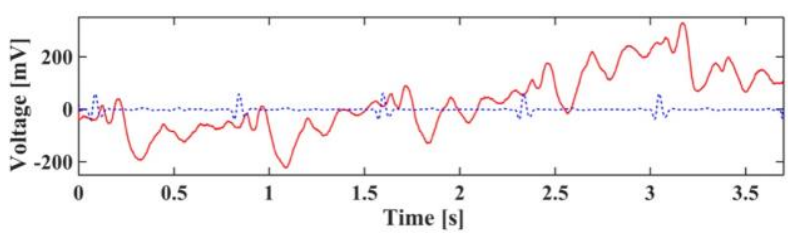

Figure 6. Measured waveform

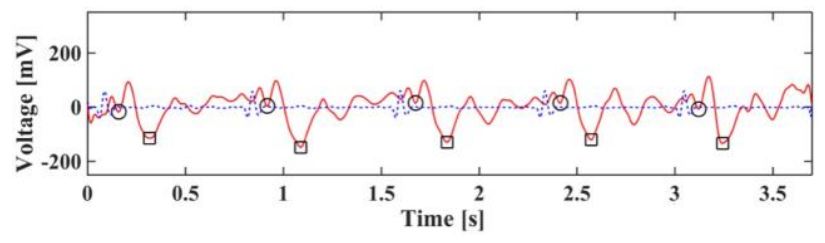

Figure 7. Filtered waveform 


\section{EXPERIMENT}

In this experiment, we verify that PWV measured by the developed device depends on systolic blood pressure. The protocol of this experiment is shown in Fig. 8, and the experiment system shown in Fig. 9. In order to vary blood pressure values, participants run on the treadmill.

Electrodes are attached to participant's both back of hands and right ankle for ECG measurement. In addition, the cuff is attached to a participant's right upper-arm for blood pressure measurement. Blood pressure is measured by the oscillometric - type sphygmomanometer (HEM7301-IT, OMRON). During the experiment, participants wear cuff in order to measure blood pressure immediately after the exercise because blood pressure decreases steadily after the exercise. A participant remains sitting in rested posture on the sensor system for 180 seconds to stabilize blood pressure. After that, his blood pressure, PWV and ECG are measured. Then, we make him run on the treadmill for 300 seconds. Speed of the treadmill is set at $7 \mathrm{~km} / \mathrm{h}$, and angle setting of the treadmill is 0 degrees. After 300 seconds, we make him sit on the device and we measure his blood pressure, PWV and ECG again.

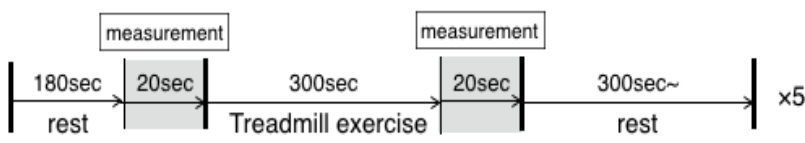

Figure 8. Experimental protocol

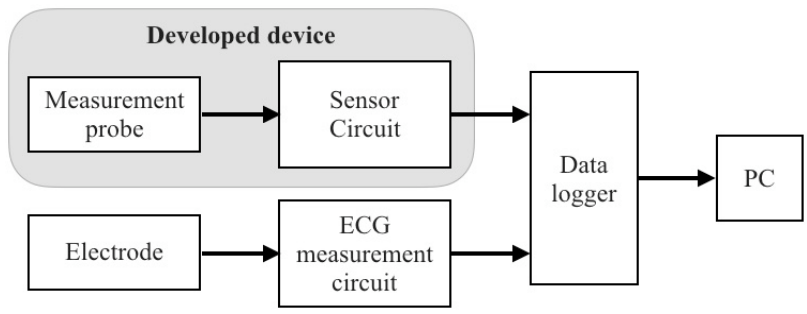

Figure 9. Schematic of the experiment system

In this experiment, this process is repeated five times. In order to reduce blood pressure value to pre-exercise value, we make him rest at least 300 seconds between each process. During measurements, participants sit on the device so that his coccyx comes in contact with the measurement probe. Participants are five men, and their age are from twenty-two to twenty-seven. Additionally, this sphygmomanometer measures systolic blood pressure when pulse wave in the process of pressurizing cuff is no longer measured. PWV and ECG are measured for 20 seconds by the system in throughout experiment because the sphygmomanometer requires 20 seconds to measure blood pressure.

If PWV values are out of the range of the average value $\pm \sigma$, those values are removed. After that, the median values of the data set are extracted as PWV value in the measurement. $\sigma$ is standard deviation value.

In order to verify the relationship between PWV which measured by our developed system and blood pressure, we evaluate the correspondence between PWV and systolic blood pressure before and after exercise.

\section{RESULTS}

Fig. 10 - 14 show relationship between square values of PWV measured by our system and systolic blood pressure measured by sphygmomanometer for each participant.

In these figures, same form of symbols shows the same trial, and white symbols show the measurement results before exercise and black ones show results after exercise. The legend is described in each figure. Moreover, the straight lines are the approximate straight lines calculated by the least square method. Equations are described in each figure.

Those figures indicate that the square value of PWV increase with the systolic blood pressure in all trials.

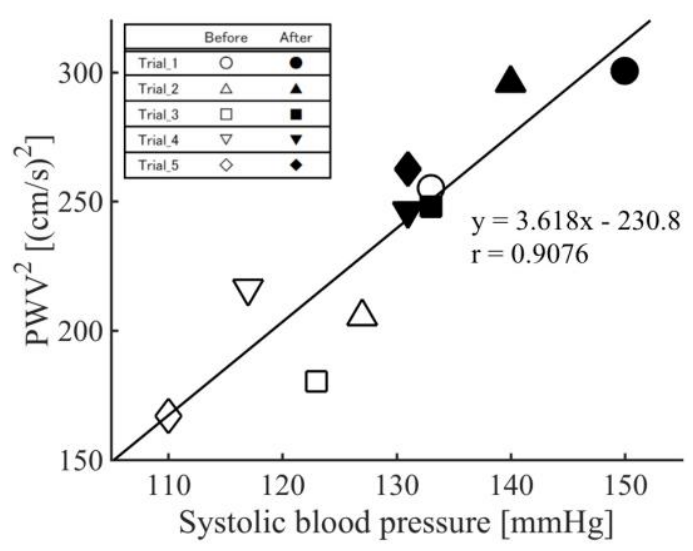

Figure 10. The result of the participant A

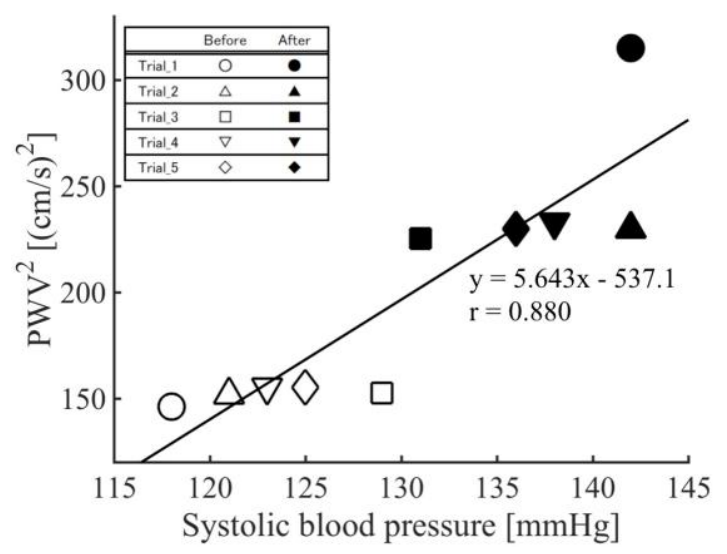

Figure 11. The result of the participant B

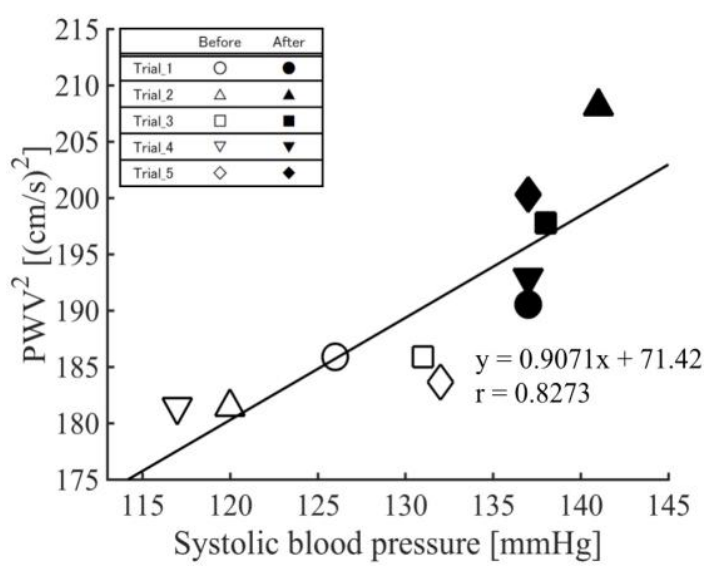

Figure 12. The result of the participant C 


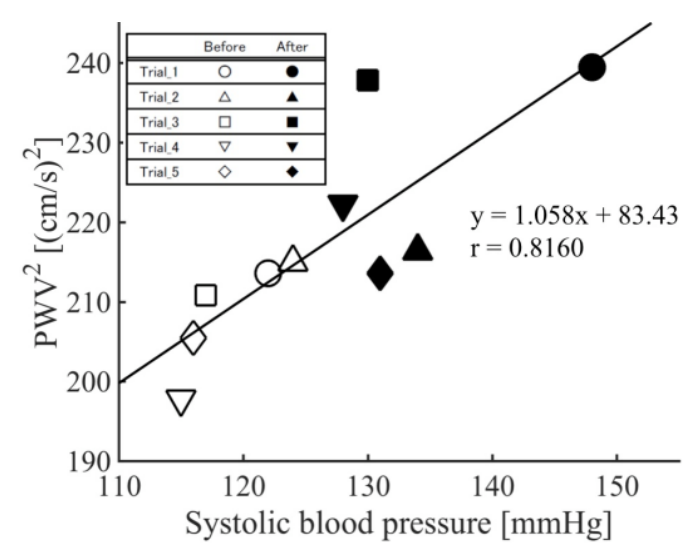

Figure 13. The result of the participant $\mathrm{D}$

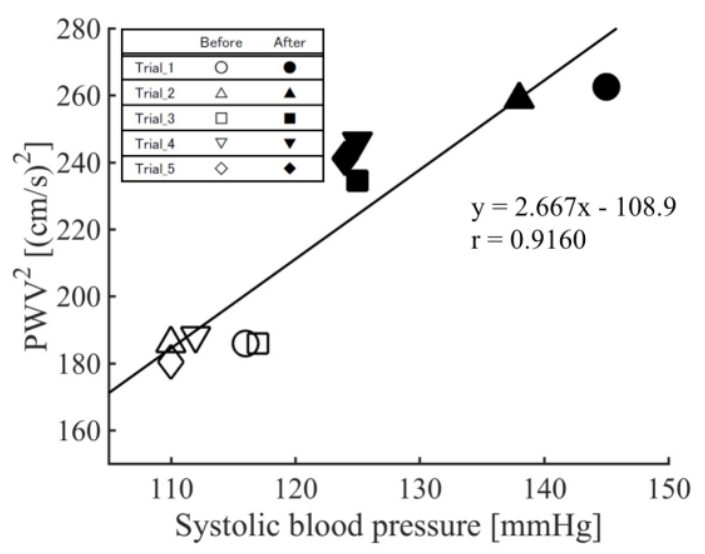

Figure 14. The result of the participant E. Some value is close to each other, therefore, some markers overlap.

We confirmed that the correlation coefficients are from 0.816 to 0.916 . The correlation coefficients are described in each figure.

The data measured from the participant E cannot apply the characteristic point extracting algorithm. Since, inflection points of $\mathrm{K}$ wave is lower than inflection points of I wave. Therefore, the rising point of $\mathrm{H}$ and the bottom point of $\mathrm{I}$ is searched backward from the inflection point of $\mathrm{K}$

\section{DISCUSSION}

The results of experiment show that there is positive correlation between the square of PWV measured by our system and the systolic blood pressure. The results of correlation coefficients are enough to imply the relationship between PWV measured by our device and blood pressure.

After the experiment, the participant E told that he has been diagnosed clockwise rotation of his heart by the doctor. It is considered that this symptom would affects the BCG waveform.

In some trials, different value of PWV was measured in spite of same systolic blood pressure. There are three main reasons about this problem. One of them is the measurement error of the sphygmomanometer. The nominal error value of this sphygmomanometer is \pm 3 $\mathrm{mmHg}$. Therefore, the measurement error would include $\pm 3 \mathrm{mmHg}$ into measured blood pressure values. In addition, that is the discord between the measurement timing of blood pressure and PWV. Blood pressure always varies. Similarly, PWV would varies constantly. Therefore, there would be a difference between the timing of sphygmomanometer to measure the systolic blood pressure and the timing of the system to calculate the median value of PWV. Third, that is the measurement error of the sensor system itself. The sensor system is susceptible to body motion. It is considered that unnecessary vibration would superimpose to BCG waveform and deform it. Therefore, wrong characteristic points are extracted.

In this study, measurement position is important to measure BCG accurately. This system measure BCG from the position of coccyx. If measurement probe touches other position, signal is attenuated by the body. Therefore, it is necessary to adjust the shape of the seat surface to the hip so as to stabilize the measurement position.

Conventional PWV measurement method requires to attach sensors to the body at least two points, however our proposed method enables to measure PWV from one point of the body. Moreover, our proposed method and system have a high affinity for human and living environment because this system is able to measure PWV without constraint. Therefore, it enables to realize comfortable health management system which does not constrain people.

\section{CONCLUSION}

In this study, we develop a novel non-restraint measurement method for measuring PWV, which relates to blood pressure strongly, and verify the relationship between the systolic blood pressure and PWV measured by the proposed method.

\section{ACKNOWLEDGMENT}

This research was funded by ImPACT Program of Council for Science, Technology and Innovation (Cabinet Office, Government of Japan).

\section{REFERENCES}

[1] S. Mendis, P. Puska, and B. Norrving, Global Atlas on Cardiovascular Disease Prevention and Control, the World Health Organization in collaboration with the World Heart Federation and the World Stroke Organization, 2011, p. 164.

[2] M. Gellman, S. Spitzer, G. Ironson, M. Llabre, P. Saab, R. D. Pasin, D. J. Weidler, and N. Schneiderman, "Poture, place, and mood effects on ambulatory blood pressure," Psychophysiology, vol. 27, no. 5, September 1990.

[3] WHO, A Global Brief on Hypertension Silent Killer, Global Public Health Crisis, WHO, 2013, pp. 19 -31.

[4] P. Fung, G. Dumont, C. Ries, C. Mott, and M. Ansermino, "Continuous noninvasive blood pressure measurement by pulse transit time," in Proc. 26th Annual International Conference of the IEEE EMBS, San Francisco, 2004, pp. 738-741.

[5] G. Lopez, M. Shuzo, H. Ushida, K. Hidaka, S. Yanagimoto, and Y. Imai, A. Kosaka, J. J. Delaunay, and I. Yamada, "Continuous blood pressure monitoring in daily life," Journal of Advanced Mechanical Design, Systems, and Manufacturing, vol. 4, no. 1, pp. 179-186, January 2010. 
[6] E. J. Kim, C. G. Park, J. S. Park, S. Y. Suh, C, U. Choi, and J. W. Kim, "Relationship between blood pressure parameters and pulse wave velocity in normotensive and hypertensive subjects: Invasive study," Journal of Human Hypertension, vol. 21, pp. 141-148, November 2006.

[7] J. C. Petrie, E. T. O’Brien, W. A. Littler, and M. de Swift, "Recommendation on blood pressure measurement," British Medical Journal, vol. 293, pp. 611-615, September 1986.

[8] K. Hayashi, T. Yamamoto, A. Takahara, and K. Shirai, "Clinical assessment of arterial stiffness with cardio - ankle vascular index: Theory and applications," Journal of Hypertension, vol. 33, no. 9, pp. 1742-1757, September 2015.

[9] E. Pinheiro, O. Postolache, and P. Girao, "Theory and developments in an unobtrusive cardiovascular system representation: Ballistocardiography," Biomedical Engineering Journal, vol. 4, pp. 201-216, October 2010.

[10] E. Saliba and Y. Sia, "The ascending aortic aneurysm: When to intervene?" IJC Heart \& Vasculature, vol. 6, no. 1, pp. 91-100, March 2015.

[11] R. K. Dishman, Y. Nakamura, M. E. Garcia, R. W. Thompson, A. L. Dunn, and S. N. Blair, "Heart rate variability, trait anxiety, and perceived stress among physically fit men and women," International Journal of Psychophysiology, vol. 37, no. 2, pp. 121133, August 2000.

[12] E. B. Kim, M. B. Susan, B. Scott, and L. B. Heddwen, Ganong's Review of Medical Physiology, New York: McGraw-Hill Education, 2012, p. 619.

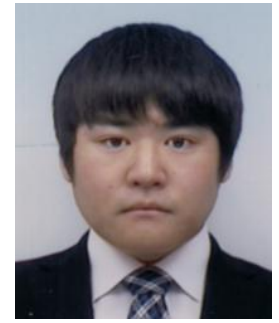

Yasunari Asakura received the B.E. degree from University of Tsukuba, Japan, in 2015. $\mathrm{He}$ is currently student of the School of Integrative and Global Majors, University of Tsukuba, Japan. His research interest is in cybernics: the fusion and combination of humans, machines and information systems.

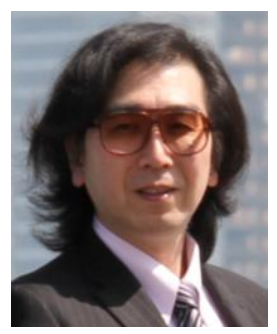

Yoshiyuki Sankai acquired a Ph.D. in Engineering from the University of Tsukuba, Japan, in 1987. He is a fellow of the Robotics Society of Japan (RSJ) (2012) and the Society of Instrument and Control Engineers (SICE) (2014). He also served as a Chairman (2005) and an Executive Board member (2004-2005) of Advanced Robotics, as well as an Executive Board member (2000-2012), congress head (2003), and President (2003) of the Japan Society of Embolus Detection and Treatment. He was a council member on the Global Agenda Councils of the World Economic Forum (20122014). He is a Professor and the Director at the Center for Cybernics Research, University of Tsukuba, the President and CEO of CYBERDYNE Inc., and a Program Manager of the Impulsing Paradigm Change through Disruptive Technologies (ImPACT) Program, initiated by the Council for Science, Technology and Innovation (CSTI) of the Cabinet Office, Japan. He has pioneered innovative cyborg-type robot technology in a new academic field, "Cybernics: the fusion and combination of humans, machines and information systems". 